\title{
Purse-string suture combined with titanium clips using a single-channel gastroscope for the closure of postintubation tracheoesophageal fistula
}
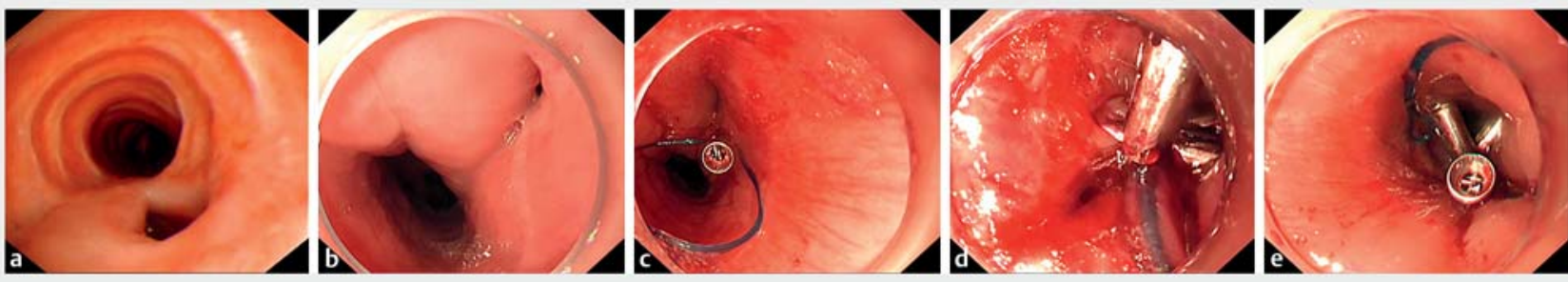

Fig. 1 Purse-string suture combined with titanium clips using a single-channel gastroscope for the closure of postintubation tracheoesophageal fistula. a Tracheoscopy showed an oval perforation measuring about $1.0 \mathrm{~cm}$ located at $20 \mathrm{~cm}$ from the nostril. b Gastroscopy revealed an approximately $1.0-\mathrm{cm}$ elongated perforation $15 \mathrm{~cm}$ away from the incisors. $\mathrm{c}$ An endoloop was inserted by forceps and anchored onto the full thickness of the edge of the fistula with clips. $\mathbf{d}$ The endoloop was tightened to pull the edges of fistula together using a removable hook. e To further strengthen the closure of the fistula, a titanium clip was added to clamp the still visible small hole at the edge of the fistula.

A 72-year-old woman was treated with endotracheal intubation and mechanical ventilation for shock caused by herbicide poisoning. After extubation she presented with cough. Tracheoscopy and gastroscopy both showed a perforation meas-

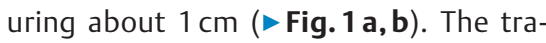
cheoesophageal fistula (TEF) was treated with a purse-string suture via a gastroscope (GIF-Q260]; Olympus, Tokyo, Japan) ( Video 1). In brief, an endoloop (Leo Medical, Changzhou, China) was inserted by forceps and anchored onto the full thickness of the edge of the fistula, including both the esophageal wall and the tracheal wall, with a titanium clip (Micro-Tech, Nanjing, China) (> Fig.1 c). After adjustment of the angle, three additional repositionable clips were used to secure the endoloop on different sides of fistula. The removable hook was then inserted and connected with the ring on the tail of the endoloop, which was tightened to pull the edges of the fistula together. The hook was then removed and the TEF was closed ( $\triangleright$ Fig. 1 d). To further strengthen the closure of the fistula, an endoclip was added to clamp the still visible small hole ( $\mathbf{F i g} \mathbf{1} \mathbf{e}$ ). The patient was put on nasal feeding after operation and her cough took a turn for the better. One month later, gastroscopy showed that the endoclips and endoloop had
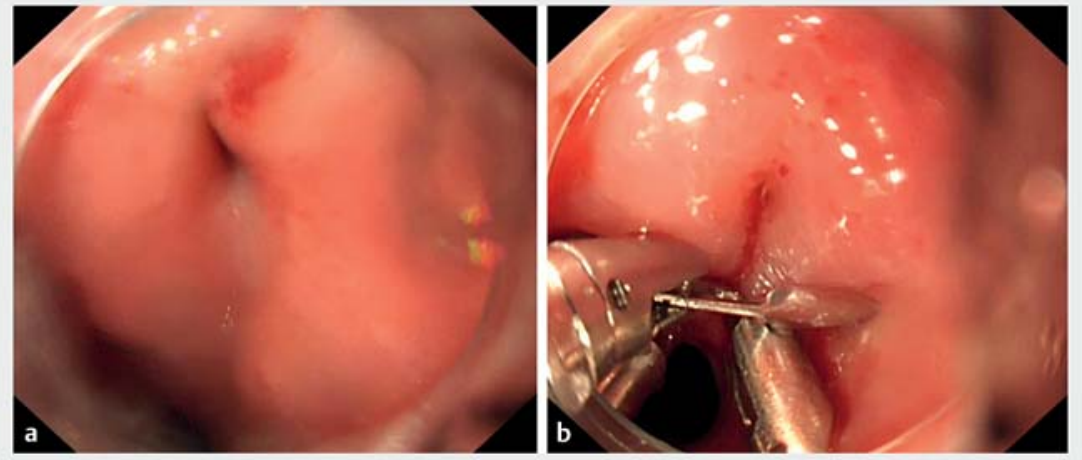

Fig. 2 Follow-up after 1 month. Gastroscopy showed that the endoclips and endoloop had fallen off and the fistula was reduced to $0.3 \mathrm{~cm}$ (a), which was clamped with three clips (b).

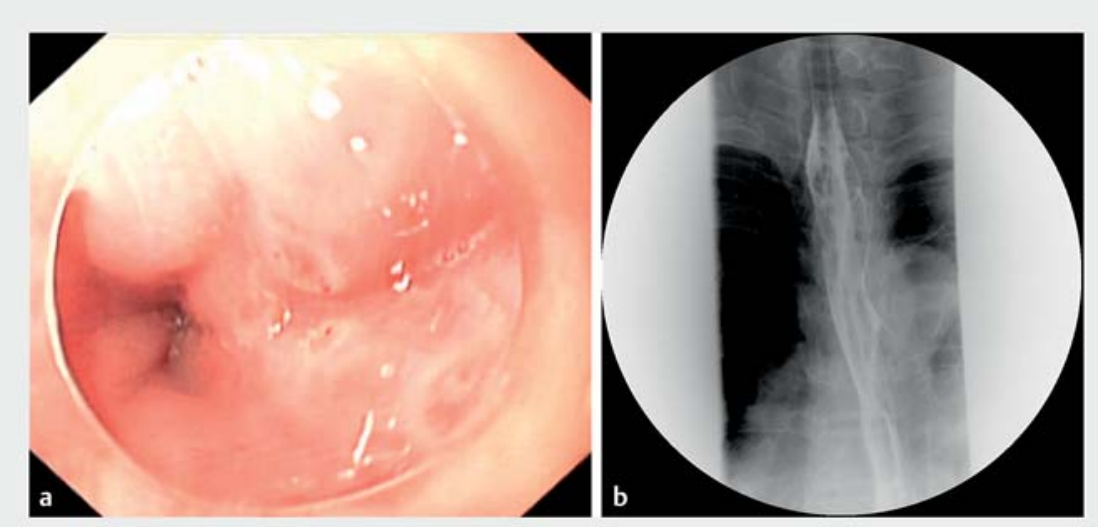

Fig. 3 Follow-up at 4 months. a Endoscopy showed a closed fistula. b Upper gastrointestinal radiography showed no extravasation of contrast medium. 


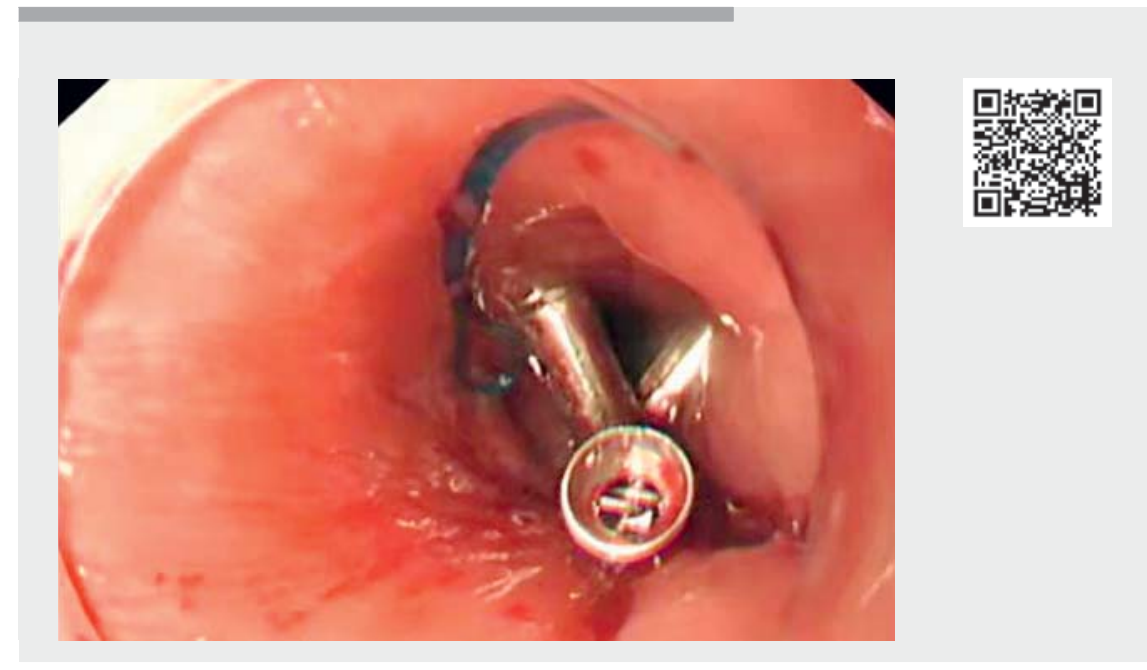

Video 1 Purse-string suture combined with titanium clips using a single-channel gastroscope for the closure of postintubation tracheoesophageal fistula in a 72-year-old woman.

\section{References}

[1] Chen W, Li J, Deng R et al. Case report: tracheoesophageal fistula secondary to postintubation tracheomegaly in a tetanus patient. Ann Palliat Med 2021; 10: 4922-4925

[2] Puma F, Vannucci J, Santoprete S et al. Surgery and perioperative management for post-intubation tracheoesophageal fistula: case series analysis. J Thorac Dis 2017; 9: 278-286

Bibliography

Endoscopy 2022; 54: E707-E708

DOI $10.1055 / \mathrm{a}-1769-4481$

ISSN 0013-726X

published online 28.2 .2022

(c) 2022. Thieme. All rights reserved.

Georg Thieme Verlag KG, Rüdigerstraße 14,

70469 Stuttgart, Germany

fallen off and the fistula was reduced to $0.3 \mathrm{~cm}$, which was clamped with three clips (\$Fig.2). Another 3 months later, gastroscopy and upper gastrointestinal radiography revealed the fistula to have closed ( $>$ Fig. 3 ).

TEF is a severe complication of endotracheal intubation [1]. Owing to the high position of postintubation TEF, it is difficult to place a tracheal or esophageal stent, and surgical correction is usually the only solution [2]. In our case, we performed a purse-string suture to reduce the fistula, and then successively closed the reduced fistula with titanium clips. This endoscopic procedure may be an alternative to surgery for postintubation TEF.

Endoscopy_UCTN_Code_TTT_1AO_2AI

\section{Competing interests}

The authors declare that they have no conflict of interest.

\section{The authors}

Shu Huang ${ }^{1}$, Sumin Zhu ${ }^{2}$, Siming Guo ${ }^{2}$, Xuan Zhao $^{2}$

1 Department of Gastroenterology, People's Hospital of Lianshui, Huaian, P. R. China

2 Department of Gastroenterology, Second Affiliated Hospital, Xuzhou Medical University, Xuzhou, P. R. China

\section{Corresponding author}

\section{Sumin Zhu, MD}

Department of Gastroenterology, Second Affiliated Hospital, Xuzhou Medical

University, 32 Meijian Road, Xuzhou 221006 , P. R. China

njzhusumin@163.com

\section{ENDOSCOPY E-VIDEOS \\ https://eref.thieme.de/e-videos}

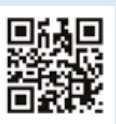

Endoscopy E-Videos is an open access online section, reporting on interesting cases and new techniques in gastroenterological endoscopy. All papers include a high quality video and all contributions are freely accessible online. Processing charges apply (currently EUR 375), discounts and wavers acc. to HINARI are available.

This section has its own submission website at

https://mc.manuscriptcentral.com/e-videos 